\title{
Deactivation of the dorsal anterior cingulate cortex indicated low postoperative sports levels in presurgical patients with chronic ankle instability
}

Xiao'ao Xue ${ }^{\dagger}$, Shengkun Li ${ }^{\dagger}$, Hongyun Li, Qianru Li and Yinghui Hua*

\begin{abstract}
Background: Injury-related fear contributed to disability in chronic ankle instability (CAI), while there still lacked exploration on the appraisal processes of the injury-related stimuli. This study aimed to compare the neural activities of the appraisal processes of sprain-related stimuli between presurgical chronic ankle instability patients and healthy controls through functional magnetic resonance imaging (fMRI) and evaluate its relationships with the clinical outcomes of orthopedic surgeries.
\end{abstract}

Methods: Eighteen presurgical CAI patients and fourteen healthy controls were recruited and underwent an fMRI session with visual stimulation of movies that showing typical ankle sprains accidents or control videos and the corresponding fear ratings. The clinical outcomes were collected at baseline and a minimum of 2 years after surgery; these included the American Orthopaedic Foot and Ankle Society (AOFAS) scores, the Numeric Rating Scale (NRS) scores, and the Tegner Activity Rating Scale scores. The two-sample t-test would be applied to identify which brain regions were influenced by CAl, and the correlation analysis would be applied to measure the relationship between the activation and clinical outcomes.

Results: Dorsal anterior cingulate cortex (dACC) was deactivated in CAl patients when compared with healthy controls, and the AACC deactivation strength revealed a moderate correlation with the values of fear ratings for all participants. The deactivation strength was negatively correlated with AOFAS at baseline, with Tegner at follow-up and its improvement.

Conclusions: Presurgical CAI patients presented deactivated dACC as a different neural activity of appraisal processes of sprain-related stimuli when compared with healthy controls, which was associated with lower postoperative sports levels. More comprehensive patients care including psychological interventions were needed in the clinical management of chronic ankle instability.

\footnotetext{
*Correspondence: hua_cosm@aliyun.com

${ }^{\dagger}$ Xiao'ao Xue and Shengkun Li contributed equally to this work and cofirst-authors

Department of Sports Medicine, Huashan Hospital, Fudan University, 12 Wulumuqi Middle Road, Shanghai 200040, China
}

\begin{abstract}
Background
The lateral ankle sprain is one of the most common sport-related injuries [1]. Over 2 million ankle sprains are treated in the US and UK each year, resulting in about $\$ 2$ billion of health-care costs [2]. In the long-term, more than $30 \%$ of patients report repetitive bouts of ankle giving way and recurring sprains, termed as chronic ankle instability (CAI) [3]. Surgical management is usually
\end{abstract} permits use, sharing, adaptation, distribution and reproduction in any medium or format, as long as you give appropriate credit to the original author(s) and the source, provide a link to the Creative Commons licence, and indicate if changes were made. The images or other third party material in this article are included in the article's Creative Commons licence, unless indicated otherwise in a credit line to the material. If material is not included in the article's Creative Commons licence and your intended use is not permitted by statutory regulation or exceeds the permitted use, you will need to obtain permission directly from the copyright holder. To view a copy of this licence, visit http://creativecommons.org/licenses/by/4.0/. The Creative Commons Public Domain Dedication waiver (http://creativeco mmons.org/publicdomain/zero/1.0/) applies to the data made available in this article, unless otherwise stated in a credit line to the data. 
suggested to treat patients who suffered serious symptoms and failed in conservative treatments to fix mechanical structures, however, some patients do not recover and fail to return to practice sports [4]. Many researchers have focused on surgical techniques and postoperative rehabilitation [5-7]. Although psychological factors have been recognized as important mediators to recovery, little is known about how they would affect clinical outcomes in patients with CAI [8].

Injury-related fear has been proposed to be one of the reasons for disability in musculoskeletal injuries [8-10]. Previous studies have suggested that patients with CAI have greater injury-related fear than healthy people, which may also lead to avoidance coping responses, such as declines in sports $[8,10,11]$. Furthermore, for the patients who had undergone surgical treatments, the appraisal of injury-related stimuli and the subsequent choices of coping strategy might also play an important role in postoperative outcomes [11-14]. According to studies on anterior cruciate ligament reconstruction, patients with a positive appraisal of the injury report a better postoperative joint function and need less time to return to practice sports compared to those patients with a passive one [15]. For example, when comparing the presurgical patients who accepted the fact of their injuries and positively participated in postoperative rehabilitation programs, and those who stuck with the fear of injuries and avoided any movements, the former ones were more likely to have less postoperative joint adhesion and muscle loss [16]. Similar research in patients with CAI is lacking, especially for the presurgical patients who suffered the influences of ankle sprains more seriously. The appraisal processes of sprain-related stimuli could be fatal for the presurgical CAI patients to get better clinical outcomes during the postoperative rehabilitation stage. Therefore, the present study is aiming at exploring the appraisal processes of sprain-related stimuli and their effect on the postoperative clinical outcomes in presurgical patients with CAI.

Functional magnetic resonance imaging (fMRI) has been widely used in psychology and neuroscience to evaluate the brain's activations in relation to human behavior [17]. As for the principle of fMRI, briefly, when a brain area is performing its function, the automatic blood regulation will surpass the oxygen consumption and decrease deoxyhemoglobin concentration, so that the localization and quantification of activated areas can be acquired by increased T2 signal, named blood oxygenation level-dependent (BOLD) signal [18]. In the present study, BOLD signal strength will be recorded as the neural activities of appraisal processes of sprain-related stimuli during the task (with visual stimulation of movies showing typical ankle sprains accidents or control videos) for the presurgical CAI patients who suffered from ankle sprains, which could be important in terms of developing more comprehensive patient care and improve the outcomes of orthopedic surgeries [15, 19-21].

Therefore, the present study had two main objectives: (1) investigating the neural activities of appraisal processes of sprain-related stimuli in presurgical CAI patients compared with healthy people, as a cross-sectional design; (2) analyzing the relationships between the central appraisal activities caused by sprain-related stimuli and postoperative outcomes in patients with CAI, as a case series design. We hypothesized that there are different neural activities of the appraisal process to the sprain-related stimuli between presurgical CAI patients and healthy people, and the strength of it would influence the surgical outcomes of patients with CAI.

\section{Methods}

The present study employed a cross-sectional fMRI design and a prospective cohort design following-up the patients' group with a minimum of 2 years postoperative. The article was composed according to the publishing guidelines of The Strengthening the Reporting of Observational Studies in Epidemiology (STROBE) statement [22]. This study has been approved by the appropriate ethics committee (Institutional Review Committee of Huashan Hospital) and has therefore been performed in accordance with the ethical standards laid down in the 1964 Declaration of Helsinki.

\section{Participants}

Between December 2016 and June 2017, 32 right-footed participants (for the consistency of functional asymmetry of brain) were recruited to participate in an fMRI study. These included 18 presurgical patients who suffered from chronic ankle instability (11 males, aged $29.0 \pm 7.2$ years; range, 17-42) and 14 healthy controls from the general community without any lower limb injury history (10 males, aged $30.6 \pm 6.5$ years; range, $23-46$ ). The patients were further followed at a minimum of 2 years after the orthopedic surgery. All participants signed written informed consent before participation.

Inclusion criteria for presurgical patients with CAI were: (1) persistent symptoms of pain, ankle giving way, and repetitive inversion sprains after 3 to 6 months of nonsurgical treatment; (2) positive Anterior Drawer Test and/or Talar Tilt Test; (3) unilaterally impaired lateral ankle ligaments, including the anterior talofibular ligament and/or the calcaneofibular ligament, confirmed by preoperative MRI $[7,23]$. All the patients were planned to accept the surgery. Exclusion criteria for both groups were as follows: (1) history of other musculoskeletal disorders or surgeries to the lower extremities; (2) history of 
cardiovascular, respiratory, neurological, autoimmune, or other major medical illnesses; (3) current usage of pharmaceuticals such as psychotropics, excitants, or betablockers; (4) people who played basketball on a regular basis due to the content of fMRI task (videos of basketball players).

\section{Part 1: task design and data analysis of fMRI}

On the day before surgery, an fMRI test was performed for both groups as the first part of this study. The task consisted of the event-designed visual stimuli that were applied to simulate the neural activities of coping response in relation to the injury-related fear [24]. Sprain stimuli (Ss) consisted of self-edited 3 s-long videos of fearful sprain accidents in basketball games with a thirdperson view, control stimuli (Cs) consisted of matched videos without sprains $[25,26]$; all videos were presented to the participants. (Fig. 1a) The intervals between stimuli were randomly varied between $0.5 \mathrm{~s}$ and $12.5 \mathrm{~s}$ [27]. Each participant performed three runs repeatedly, and each run was composed of 8 Ss and $8 \mathrm{Cs}$ that presented in a pseudorandom manner (the orders of the stimuli were generated randomly before the study and kept the same for all participants). During each stimulus, participants were asked to imagine how they would feel if that situation occurred to them and then rate their feelings immediately through an MR-compatible assessment responder. There were three rating options: afraid $(-1)$, neutral $(0$, no response) or pleasant $(+1)$ so that the fear levels induced by two kinds of stimuli could be evaluated by the average rating scores, and the levels of injury-related fear could be calculated by difference values quantitatively [28] (Fig. 1b).
A 3.0-T Siemens Magnetom Verio scanner equipped with an 8-channel head coil was used for scanning. Detailed data acquisition and image preprocessing steps are provided in the Additional file 1. For the firstlevel statistical analysis, Statistical Parametric Mapping (SPM8, Department of Cognitive Neurology, London) that implemented in MATLAB version R2014a (The MathWorks Inc., Natick, Massachusetts) was used. A general linear model was specified by SPM8 for the Ss conditions, Cs conditions, and rest conditions according to time series. BOLD signal strength (beta value) of each condition were obtained, convolved with the canonical hemodynamic response function and high-pass filtered $(1 / 128 \mathrm{~Hz})$ to remove low-frequency noise caused by scanner signal drifts [29]. For the second-level statistical analysis, Restplus Version 1.22 (http://restfmri.net/ forum/index.php?q=rest) was used. Contrast images for "Ss condition > rest condition", "Cs condition $>$ rest condition" and "Ss condition $>$ Cs condition" for each participant were calculated to reveal neural activations caused by the two kinds of stimulations and the sprain-related stimuli without basketball playing scene. The contrast images of "Ss condition > Cs condition" were compared between groups using a two-sample t-test to identify which brain regions had significant differences in neural activations of appraisal processes of the visual presented sprain-related stimuli. The multivariable regression was also repeated to control age and sex. The two-tailed cluster-level threshold of $p<0.05$ and voxel-level threshold of $p<0.001$ with Gaussian Random Field corrections were used to define significance.

The areas showing significant differences were saved as masks and the signal strength within them was extracted from the contrast images of "Ss condition $>$ Cs condition"
(A)
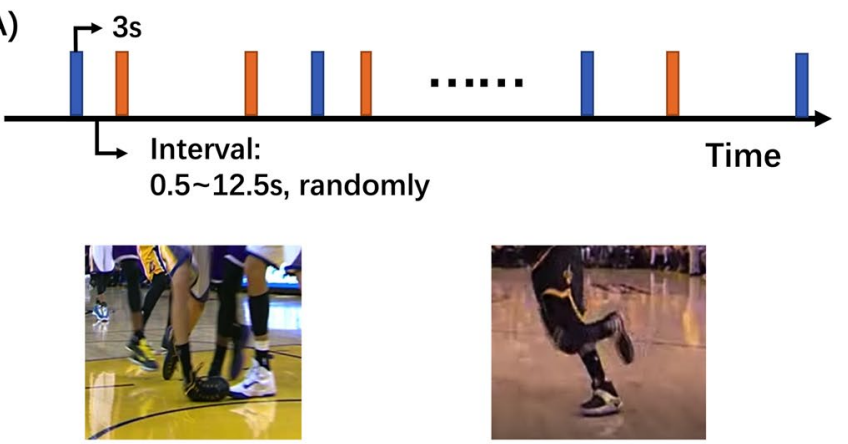

SPRAIN stimulation

(Ss)

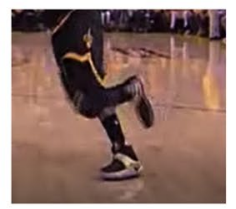

CONTROL stimulation (Cs)
(B)

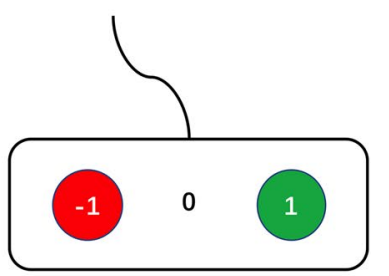

1 Afraid

0 Neutral

-1 Pleasant

Fig. 1 a Each run was composed of 8 SPRAIN stimuli of sprain accident and 8 CONTROL stimuli of basketball playing presented in a pseudorandom manner; b An MR-compatible assessment responder with three rating options: afraid $(-1)$, neural $(0$, no response) or pleasant $(+1)$, to measure the degree of fear induced by each stimulus 
for each participant to perform further analyses. If the signal strength was negative, a positive-negative switch would be displayed and defined as deactivation strength to facilitate analysis and discussion.

\section{Part 2: treatment procedure and clinical evaluation}

All patients were treated in our hospital, and the arthroscopic ankle stabilization surgeries were performed by a senior surgeon (the corresponding author) according to previously described standardized methods [5, 30]. Briefly, the lateral ligament repair would be considered if the ATFL remnant was sufficient and in good condition, or anatomical reconstruction with semitendinosus allograft or gracilis autograft would be performed [5, 30]. All patients were encouraged to walk without support if they were able to, and they all accepted standardized postoperative rehabilitation after the operation. For the second part of this study, patients were followed-up at a minimum of 2 years after surgery, which was long enough for them to get satisfactory postoperative outcomes according to our previous studies. Clinical evaluations, including (1) the 100-points American Orthopaedic Foot and Ankle Society (AOFAS) score for ankle function, which measured the severity and frequency of pain (40 points), activity limitations/support requirement (10 points), maximum walking distance (5 points), walking surfaces (5 points), gait abnormality (8 points), sagittal motion (8 points), hindfoot motion (6 points), ankle-hindfoot stability (8 points), and alignment of ankle-hindfoot (10 points); (2) the 10-point Numeric Rating Scale (NRS) for subjective severity of current pain, from "no pain" (0 points) to "unbearable pain" (10 points); (3) and the 10-point Tegner Activity Rating Scale (Tegner) score for sports level, form "sick leave or disability pension because of the joint problem" (0 points) to "Competitive sports Soccer-national and international elite" (10 points), were administered at baseline for both groups and re-administered for the patient's group. Improvements in scores would be calculated as the difference in values between the clinical scores at the two-time points. The time of return to work after surgery was also measured. All evaluations were performed by a blinded physician.

\section{Statistical analysis}

Statistical analysis was performed in Graphpad Prism Version 8.0 (GraphPad Software, San Diego, California). Descriptive statistics were calculated as mean \pm standard deviation. Mann-Whitney U tests, Chi2 tests, and 2-sample t-tests were performed to compare demographic variables between groups. Paired t-tests or Wilcoxon tests were performed to compare clinical scores at baseline and follow-up in the patient's group. The fear ratings under each condition were analyzed by group-level ANOVA with pair-wise Bonferroni multiple comparison tests. Correlations between signal strength and clinical scores were determined by Pearson or Spearman correlation coefficients. The selections of parametric or non-parametric tests were based on variance homogeneity and normality of the data. The statistical significance level was set at 0.05 . Participants with excessive head motions (more than $3 \mathrm{~mm}$ or $3^{\circ}$ ) in the first part and patients who dropped out from the follow-up or reinjured in the second part were excluded [31].

\section{Results}

Demographic and clinical features

A total of 13 healthy controls and 16 presurgical CAI patients were enrolled in the first part of this study (1 healthy control and 2 patients were excluded due to excessive head motion). Among the patients, 11 underwent the repair and 5 underwent the reconstruction $(2$ with allograft and 3 with autograft) of the lateral ligaments. There were no significant differences between the two groups in all demographic features $(p>0.05)$, while significant differences were found in the AOFAS, NRS and Tegner score $(p<0.01)$ between two groups, indicating worse ankle functions, pain levels and sports participation in the patient group at baseline (Table 1).

\section{Part 1: fMRI tests on the central Appraisal response to the fear}

For the fMRI evaluation, one cluster within "Ss condition $>$ Cs condition" contrast was significantly deactivated in patients with CAI when compared with healthy controls. The cluster was located in Brodmann's area 24 and 32 , defined as the dorsal anterior cingulate cortex (dACC). The size of the cluster was 350 voxels,

Table 1 Demographic variables of control group and patient group

\begin{tabular}{|c|c|c|c|c|c|}
\hline & \multicolumn{2}{|c|}{$\begin{array}{l}\text { Control group } \\
(n=13)\end{array}$} & \multicolumn{2}{|c|}{$\begin{array}{l}\text { Patients group } \\
(n=16)\end{array}$} & \multirow[t]{2}{*}{$p$ value } \\
\hline & Mean & SD & Mean & SD & \\
\hline Age (years) & 29.5 & 5.1 & 28.6 & 6.8 & 0.71 \\
\hline Weight (kg) & 68.6 & 11.9 & 62.7 & 10.1 & 0.17 \\
\hline Height (m) & 1.73 & 0.08 & 1.70 & 0.10 & 0.41 \\
\hline Education level (years) & 16.0 & 1.2 & 15.0 & 2.2 & 0.15 \\
\hline Male/female & $10 / 3$ & & $10 / 6$ & & 0.45 \\
\hline Duration (months) & - & - & 37.5 & 33.9 & - \\
\hline AOFAS & 100.0 & 0.0 & 73.7 & 10.8 & $<0.01$ \\
\hline NRS & 0.0 & 0.0 & 3.3 & 1.5 & $<0.01$ \\
\hline Tegner & 6.2 & 0.6 & 3.8 & 1.0 & $<0.01$ \\
\hline
\end{tabular}

AOFAS, American Orthopaedic Foot and Ankle Society score; NRS, Numeric Rating Scale; Tegner, Tegner Activity Rating Scale 
the peak MNI coordinate was $(8,26,24)$, and the intensity ( $\mathrm{t}$ value) was -4.58 . (Fig. $2 \mathrm{a}$ ). A positive-negative switch was performed to the signal strength of "Ss condition > Cs condition" contrast images as the deactivation strength. When comparing fear rating between groups, no significant difference under Cs $(p>0.99$, $95 \% \mathrm{CI}-0.61$ to 0.41$)$ and greater fear levels in patients under Ss $(p<0.01,95 \%$ CI 0.23 to 1.00$)$ were observed. (Fig. 2b) The correlation between dACC deactivation strength and the difference in values of fear ratings (between Ss and Cs) revealed a moderate correlation $(p=0.04, \mathrm{r}=-0.381,95 \%-0.66$ to -0.02$)$ for all participants (Fig. 2c).
Part 2: relationships between $\mathrm{fMRI}$ signals and clinical outcomes

Median follow-up time was 34 (ranged from 30 to 35) months for the patients in the second part of this study. One patient (reconstruction with autograft) who dropped out from follow-up and two patients (repair) who suffered from another ankle sprain accident after the surgery were excluded. None of the remaining patients had further ankle sprains or other orthopedic injuries. All clinical outcomes, including AOFAS $(p<0.01)$, NRS $(p<0.01)$, and Tegner scores $(p<0.01)$, were significantly improved at follow-up. The average time to return to work was $7.0 \pm 1.7$ weeks.

(a)

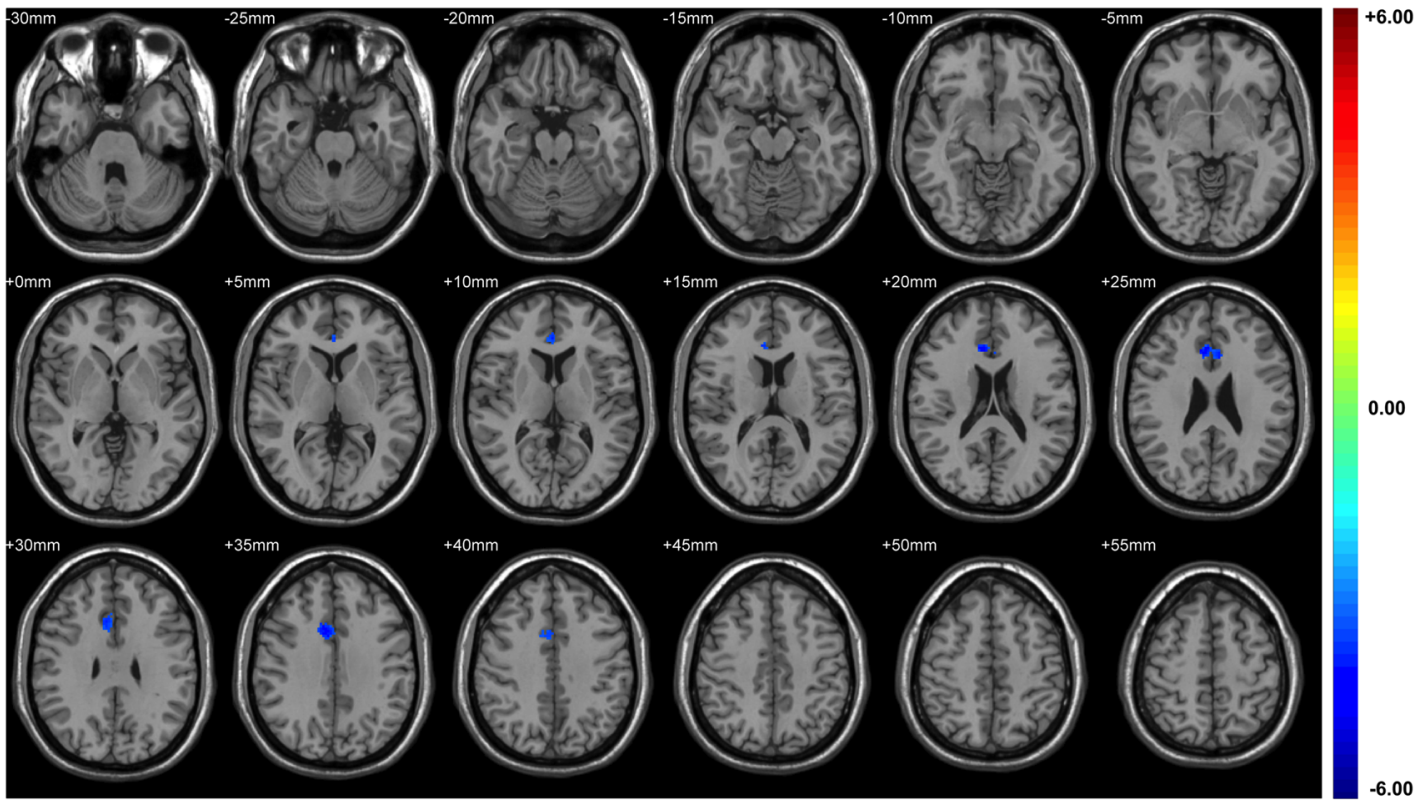

(b)

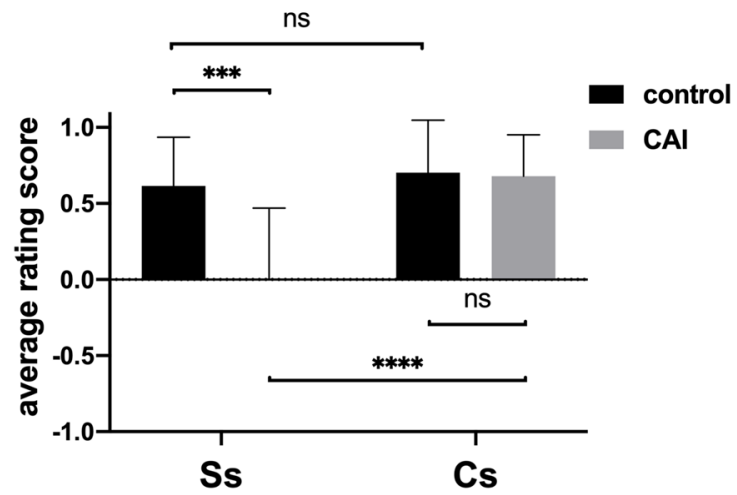

(c)

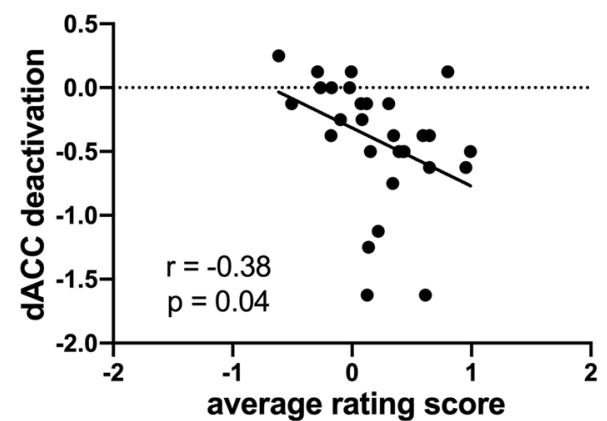

Fig. 2 a The result of two sample t test of contrast images of "Ss condition>Cs condition" (SPRAIN stimuli minus CONTROL stimuli) between Patient Group and Control Group after two-tailed Gaussian Random Field correction with the cluster-level threshold of $p<0.05$ and voxel-level threshold of $p<0.001 ; \mathbf{b}$ the comparison of average rating scores between two groups under two stimuli after pair-wise Bonferroni multiple comparison tests; $\mathbf{c}$ the correlation between dACC deactivation strength and difference values of fear ratings; ns, no significant difference; ${ }^{*}$ adjusted $p<0.05 ; *^{* *}$ adjusted $p<0.01 ; * *$ adjusted $p<0.001 ; * * *$ adjusted $p<0.0001$ 
To investigate the relationship between dACC deactivation strength and durations, time to return to work, clinical scores at baseline and follow-up, and the improvements of clinical scores, a set of correlation analyses were performed. At the baseline, AOFAS was significantly negatively correlated with the deactivation strength $(\mathrm{r}=-0.60, p=0.03)$. At follow-up, the postoperative Tegner score $(r=-0.59, p=0.04)$ was found to be significantly negatively correlated with deactivation strength. For the improvement, the deactivation strength was shown to be significantly correlated (positively) with the AOFAS $(\mathrm{r}=0.80, p<0.01)$ and (negatively) with the Tegner improvement $(\mathrm{r}=-0.66, p=0.02)$. A trend of correlation between deactivation strength and time to return to work was also observed $(\mathrm{r}=0.55, p=0.05)$. No other significant correlations were identified $(p>0.05)$, (Table 2).

\section{Discussion}

The most important finding of this study was the deactivation of $\mathrm{dACC}$ as a different appraisal process of the sprain-related stimuli in presurgical patients with CAI when compared with healthy people, which was also associated with lower postoperative sports level. To the best of our knowledge, the present study is the first fMRI study on the evaluation of appraisal processes of the sprain-related stimuli and its relationship with surgical outcomes in patients with CAI.

Numerous studies have demonstrated the role that injury-related fear plays in the disability of musculoskeletal diseases $[8,10,32-36]$. However, all of the past and recent studies on injury-related fear in patients with CAI have used self-reported evaluation of fear and avoidance and lacked a further exploration of the neural activities of its appraisal processes. To overcome this limitation and advance the knowledge in this area the present study used fMRI to explore the neural activities of its appraisal processes of the injury-related stimuli quantitatively [17]. Comparing the whole brain activation, a significant deactivation within dACC was observed under visual presented sprain-related stimuli in patients with CAI compared with healthy controls, in this study.

The dorsal anterior cingulate cortex is a part of the limbic system located in the inner side of the cerebral hemispheres and associated with the appraisal of fear-related stimuli [24, 37]. However, increased activity of dACC has commonly been observed in previous studies on fear emotion, which seemed to be contrary to our results [28, 38]. The explanation for that may be due to differences in the study designs. Previous studies aimed to evaluate the expression of fear, and therefore, stimuli with low cognitive load (such as fearful pictures) were presented to induce conditioned fear, leading to increased activation of the dACC $[24,38]$. In the present study, we aimed to simulate the appraisal process of the sprain accidents, and therefore, self-imaginations with high cognitive load were induced when watching the sprain accident videos, which may have resulted in the deactivation of the dACC [25]. A previous study on shoulder apprehension with a similar task design to the present study also observed the deactivation of the dACC [25]. In addition, regarding its positive correlation with fear levels, this change may represent a passive appraisal process of sprain-related stimuli and be associated with injury-related fear of the patient with CAI $[11,21,39]$.

To further explore the clinical meanings of this appraisal process in patients with CAI, the relationship between dACC deactivation strength and clinical outcomes were evaluated. The significant correlation with the AOFAS score at baseline suggested that worse ankle function was associated with a higher passive appraisal process of sprain-related stimuli. The experience of serious symptoms and recurrent sprains in patients might increase their appraised degrees of the threat caused

Table 2 Correlations of dACC deactivation strength with clinical outcomes in patients of baseline, 3 years follow-up and their improvements

\begin{tabular}{|c|c|c|c|c|c|c|c|c|c|}
\hline \multirow[t]{2}{*}{ dACC deactivation } & \multicolumn{3}{|c|}{ Baseline } & \multicolumn{3}{|c|}{ Follow-up } & \multicolumn{3}{|c|}{ Improvement } \\
\hline & $r$ & $95 \% \mathrm{Cl}$ & $p$ & $\mathbf{r}$ & $95 \% \mathrm{Cl}$ & $p$ & $r$ & $95 \% \mathrm{Cl}$ & $p$ \\
\hline AOFAS $^{\mathrm{a}}$ & $-0.60^{*}$ & -0.87 to -0.08 & 0.03 & 0.04 & -0.52 to 0.58 & 0.89 & $0.80^{* *}$ & 0.44 to 0.94 & $<0.01$ \\
\hline $\mathrm{NRS}^{\mathrm{b}}$ & 0.33 & -0.29 to 0.75 & 0.28 & -0.04 & -0.59 to 0.54 & 0.90 & -0.33 & -0.76 to 0.28 & 0.27 \\
\hline Tegner $^{b}$ & -0.14 & -0.65 to 0.46 & 0.65 & $-0.59^{*}$ & -0.86 to -0.03 & 0.04 & $-0.66^{*}$ & -0.89 to -0.15 & 0.02 \\
\hline Return to work (weeks) & - & & & $0.55^{\wedge}$ & 0.002 to 0.85 & 0.05 & - & & \\
\hline Duration $^{\mathrm{a}}$ & -0.10 & -0.61 to 0.48 & 0.76 & - & & & - & & \\
\hline
\end{tabular}

dACC, Dorsal Anterior Cingulate Cortex; AOFAS, American Orthopaedic Foot and Ankle Society score; NRS, Numeric Rating Scale; Tegner, Tegner Activity Rating Scale

a Pearson correlation coefficient

${ }^{\mathrm{b}}$ Spearman correlation coefficient

${ }^{\wedge} p=0.05 ;{ }^{*} p<0.05 ;{ }^{* *} p<0.01$ 
by ankle sprains, induced more passive emotions (e.g. injury-related fear) and the corresponding avoidance coping of movements (especially the rehabilitation training), which could also make their ankle functions worse $[11,14,40]$. Furthermore, this change might depend more on severity than the time length of CAI, as supported by its non-significant correlation with the duration of symptoms. At a minimum follow-up of 2 years, the significant correlations between dACC deactivation and postoperative Tegner scores and its improvement fitted the previous evidence that the passive appraisal process and its corresponding avoidance coping response would become maladaptive in the long term recovery, making patients turning away from risky behaviors to avoid their fear of injuries (such as postoperative rehabilitation) $[15,21,39]$. A trend of correlation with the time to return to work also supported this view, as returning to sports or heavy physical activities might not be a priority for patients from community anymore, differently in comparison to previous studies on athletes who lived on sports $[12,13,41]$. Although in contrast with previous findings, we observed that patients with higher dACC deactivation reported a higher improvement in ankle function [15, 21]. A potential reason for that, as supported by the Tegner scores could be that the avoidance of heavy physical activity facilitated a reduction in residual symptoms postoperatively. Although previous studies indicated that $\mathrm{AACC}$ contributed to pain processing, the non-significant result in our study suggested that the deactivated dACC might not play an important role in pain among patients with CAI in this study [42].

We intended to investigate the mechanisms underpinning the appraisal processes of the injury-related stimuli in this study, because our clinical experience suggested that some postoperative patients, were extremely worried about the condition of their ankle and preferred to avoid movement after ankle orthopedic surgeries. Our findings provide further insight into the appraisal processes of the injury-related stimuli in patients with CAI and emphasized its important role in both progression and postoperation stages. This in turn might shift the attention to psychological factors and, hopefully, promote the development of more specific psychological interventions on passive appraisal processes of the injury-related stimuli in order to improve the surgical outcomes for patients with CAI and even other sports injuries in the future.

This study was not without limitations. First, the sample size was relatively small, however, it was similar to other studies with similar protocol $[25,28]$. More multivariable regressions or subgroup analyses in a larger sample might explain the role of appraisal processes of the sprain-related stimuli in presurgical patients with CAI better. Next, the current study only performed the fMRI tests at baseline due to the difficulty in recalling patients back from all over the country. A more detailed longitudinal study on how the surgery (e.g. repair or reconstruction) or rehabilitation influenced AACC activation should be conducted in future studies. Then, this study only focused on the fMRI test of neural activities with fear rating, and the lack of standardized self-reported questionnaires on the emotional responses and coping procedure of the CAI patients before/after the surgery did reduce the reliability of this study. Finally, the patients enrolled in this study were not fully in line with the recommendation of the International Ankle Consortium. However, as this study focused on the surgical outcomes, our inclusion may represent the presurgical patients with serious symptoms and mechanical instability better than the recommended one.

\section{Conclusions}

Our study suggested that presurgical CAI patients presented deactivated $\mathrm{dACC}$ as a different neural activity of appraisal processes of on sprain-related stimuli when compared with healthy controls, and the higher deactivation strength was associated with lower postoperative sports levels. As the psychological factors might influence the outcome of orthopedic surgeries, more comprehensive patients cares including psychological interventions are needed in the clinical management of presurgical patients with CAI.

\section{Abbreviations}

CAl: Chronic ankle instability; dACC: Dorsal Anterior Cingulate Cortex; fMRI: Functional magnetic resonance imaging; BOLD: Blood oxygenation leveldependent; AOFAS: The American Orthopaedic Foot and Ankle Society scores; NRS: The Numeric Rating Scale scores; Ss: Sprain stimuli; Cs: Control stimuli.

\section{Supplementary Information}

The online version contains supplementary material available at https://doi. org/10.1186/s13102-021-00353-6.

Additional file 1. Detailed fMRI data acquisition and preprocessing steps.

\section{Acknowledgements}

The authors gratefully acknowledge the help in fMRI data preprocessing of Zhiyuan, Liu from Shanghai Key Laboratory of Magnetic Resonance and Department of Physics, East China Normal University, Shanghai, China, and enlightened education of fMRI for Xiao'ao Xue of Prof. Yufeng Zang and Xize Jia from Institutes of Psychological Sciences, Hangzhou Normal University, Hangzhou, China. The authors would also like to express their gratitude to EditSprings (https://www.editsprings.com/) for the expert linguistic services provided.

\section{Authors' contributions}

XX managed conceptualization, methodology, formal analysis, and writing original draft. SL managed validation, data collection, methodology and visualization. $\mathrm{HL}$ and QL managed investigation. YH managed manuscript review \& editing, project administration, and funding acquisition. XX and SL contributed equally to this study and were co-first-authors. 


\section{Funding}

This work was supported by the National Natural Science Foundation of China (Grant No. 81871823) and the Science and Technology Commission of Shanghai Municipality (Grant No. 18JC1410403).

\section{Availability of data and materials}

The original imaging data used to support the findings of this study have not been made available because of the patient privacy, while the extracted data and the mask of the significant cluster are available from the corresponding author upon request.

\section{Declarations}

\section{Ethics approval and consent to participate}

This study have been approved by the Institutional Review Committee of Huashan Hospital (reference No.2016314) and have therefore been performed in accordance with the ethical standards laid down in the 1964 Declaration of Helsinki. Informed consent was obtained from all individual participants included in the study.

\section{Consent for publication}

Not applicable.

\section{Competing interests}

The authors declare that they have no competing interests.

Received: 27 March 2021 Accepted: 30 September 2021 Published online: 09 October 2021

\section{References}

1. Fong DTP, Hong Y, Chan LK, et al. A systematic review on ankle injury and ankle sprain in sports. Sport Med. 2007;37:73-94.

2. Soboroff SH, Pappius EM, Komaroff AL. Benefits, risks, and costs of alternative approaches to the evaluation and treatment of severe ankle sprain. Clin Orthop Relat Res. 1984;183:160-8.

3. Anandacoomarasamy A, Barnsley L. Long term outcomes of inversion ankle injuries. Br J Sports Med. 2005;39:e14.

4. Doherty C, Bleakley C, Delahunt E, et al. Treatment and prevention of acute and recurrent ankle sprain: an overview of systematic reviews with meta-analysis. Br J Sports Med. 2017:51:113-25.

5. Hua $Y, C$ Chen $S$, Jin $Y$, et al. Anatomical reconstruction of the lateral ligaments of the ankle with semitendinosus allograft. Int Orthop. 2012;36:2027-31

6. Tsikopoulos K, Mavridis D, Georgiannos D, et al. Does multimodal rehabilitation for ankle instability improve patients' self-assessed functional outcomesa network meta-analysis. Clin Orthop Relat Res. 2018;476:1295-310.

7. Song Y, Li H, Sun C, et al. Clinical guidelines for the surgical management of chronic lateral ankle instability: a consensus reached by systematic review of the available data. Orthop J Sport Med. 2019. https:// doi.org/10.1177/2325967119873852.

8. Houston M, Hoch J, Hoch M. Collegiate athletes with ankle sprain history exhibit increased fear-avoidance beliefs. J Sport Rehabil. 2017;49:A16.2-A16.

9. Wertli MM, Rasmussen-Barr E, Weiser S, et al. The role of fear avoidance beliefs as a prognostic factor for outcome in patients with nonspecific low back pain: a systematic review. Spine J. 2014;14:816-836.e4.

10. Houston MN, Van Lunen BL, Hoch MC. Health-related quality of life in individuals with chronic ankle instability. J Athl Train. 2014;49:758-63.

11. Lazarus RS, Folkman S. Stress, appraisal, and coping. New York: Springer; 1984.

12. LentzTA, Zeppieri G, George SZ, et al. Comparison of physical impairment, functional, and psychosocial measures based on fear of reinjury/ lack of confidence and return-to-sport status after ACL reconstruction. Am J Sports Med. 2015;43:345-53.

13. Everhart JS, Best TM, Flanigan DC. Psychological predictors of anterior cruciate ligament reconstruction outcomes: a systematic review. Knee Surgery, Sport Traumatol Arthrosc. 2015;23:752-62.
14. Lazarus RS. Psychological stress and the coping process. New York: McGraw-Hill; 1966.

15. Burland JP, Toonstra JL, Howard JS. Psychosocial barriers after anterior cruciate ligament reconstruction: a clinical review of factors influencing postoperative success. Sports Health. 2019;11:528-34.

16. Hagger MS, Griffin M, Chatzisarantis NLD, et al. Injury representations, coping, emotions, and functional outcomes in athletes with sportsrelated injuries: a test of self-regulation theory. J Appl Soc Psychol. 2005:35:2345-74.

17. Schilaty ND, Nagelli C, Hewett TE. Use of objective neurocognitive measures to assess the psychological states that influence return to sport following injury. Sport Med. 2016;46:299-303.

18. Gauthier CJ, Fan AP. BOLD signal physiology: models and applications. Neuroimage. 2019;187:116-27.

19. Waddell G. A Fear-Avoidance Beliefs Questionnaire (FABQ) and the role of fear-avoidance beliefs in chronic low back pain and disability Gordon. Pain. 1993;29:465-9.

20. Heijne A, Axelsson K, Werner S, et al. Rehabilitation and recovery after anterior cruciate ligament reconstruction: patients' experiences. Scand J Med Sci Sport. 2008:18:325-35.

21. Carson F, Polman RCJ. The facilitative nature of avoidance coping within sports injury rehabilitation. Scand J Med Sci Sport. 2010;20:235-40.

22. Von Elm E, Altman DG, Egger M, et al. The Strengthening the Reporting of Observational Studies in Epidemiology (STROBE) statement: guidelines for reporting observational studies. PLoS Med. 2007;4:1623-7.

23. Li H, Hua Y, Feng S, et al. Lower signal intensity of the anterior talofibular ligament is associated with a higher rate of return to sport after ATFL repair for chronic lateral ankle instability. Am J Sports Med. 2019:47:2380-5.

24. Kalisch R, Wiech K, Critchley HD, et al. Levels of appraisal: a medial prefrontal role in high-level appraisal of emotional material. Neuroimage. 2006;30:1458-66.

25. Haller S, Cunningham G, Laedermann A, et al. Shoulder apprehension impacts large-scale functional brain networks. Am J Neuroradiol. 2014;35:691-7

26. Barke A, Baudewig J, Schmidt-Samoa C, et al. Neural correlates of fear of movement in high and low fear-avoidant chronic low back pain patients: an event-related fMRI study. Pain. 2012;153:540-52.

27. Burock MA, Buckner RL, Woldorff MG, et al. Randomized event-related experimental designs allow for extremely rapid presentation rates using functional MRI. NeuroReport. 1998;9:3735-9.

28. Shitara H, Shimoyama D, Sasaki T, et al. The neural correlates of shoulder apprehension: a functional MRI study. PLOS ONE. 2015. https://doi.org/10. 1371/journal.pone.0137387.

29. Friston KJ, Worsely KJ. Analysis of fMRI time-series revisited-again. Neuroimage. 1995;2:173-81.

30. Li H, Hua Y, Li H, et al. Activity level and function 2 years after anterior talofibular ligament repair: a comparison between arthroscopic repair and open repair procedures. Am J Sports Med. 2017:45:2044-51.

31. Friston KJ, Williams S, Howard R, et al. Movement-related effects in fMRI time-series. Magn Reson Med. 1996:35:346-55.

32. Simon CB, Stryker SE, George SZ. Comparison of work-related fear-avoidance beliefs across different anatomical locations with musculoskeletal pain. J Pain Res. 2011;4:253-62.

33. Mintken PE, Cleland JA, Whitman JM, et al. Psychometric properties of the fear-avoidance beliefs questionnaire and Tampa Scale of Kinesiophobia in patients with shoulder pain. Arch Phys Med Rehabil. 2010;91:1128-36.

34. Wikstrom EA. Fear of re-injury does not differ between those with and without chronic ankle instability. J Sport Sci Med. 2011;10:771-2.

35. Woby SR, Roach NK, Urmston M, et al. Psychometric properties of the TSK-11: a shortened version of the Tampa Scale for Kinesiophobia. Pain. 2005:117:137-44.

36. Lentz TA, Sutton Z, Greenberg S, et al. Pain-related fear contributes to selfreported disability in patients with foot and ankle pathology. Arch Phys Med Rehabil. 2010;91:557-61.

37. Heilbronner SR, Hayden BY. Dorsal anterior cingulate cortex: a bottom-up view. Annu Rev Neurosci. 2016;39:149-70.

38. Mechias ML, Etkin A, Kalisch R. A meta-analysis of instructed fear studies: implications for conscious appraisal of threat. Neuroimage. 2010:49:1760-8. 
39. Podlog L, Heil J, Schulte S. Psychosocial factors in sports injury rehabilitation and return to play. Phys Med Rehabil Clin N Am. 2014;25:915-30

40. Needle AR, Lepley AS, Grooms DR. Central nervous system adaptation after ligamentous injury: a summary of theories, evidence, and clinical interpretation. Sport Med. 2017:47:1271-88.

41. Kvist J, Ek A, Sporrstedt K, et al. Fear of re-injury: a hindrance for returning to sports after anterior cruciate ligament reconstruction. Knee Surgery, Sport Traumatol Arthrosc. 2005;13:393-7.
42. Xiao $X$, Zhang $Y Q$. A new perspective on the anterior cingulate cortex and affective pain. Neurosci Biobehav Rev. 2018;90:200-11.

\section{Publisher's Note}

Springer Nature remains neutral with regard to jurisdictional claims in published maps and institutional affiliations.
Ready to submit your research? Choose BMC and benefit from:

- fast, convenient online submission

- thorough peer review by experienced researchers in your field

- rapid publication on acceptance

- support for research data, including large and complex data types

- gold Open Access which fosters wider collaboration and increased citations

- maximum visibility for your research: over $100 \mathrm{M}$ website views per year

At BMC, research is always in progress.

Learn more biomedcentral.com/submissions 